\title{
DIE NEUE VERFASSUNG DER VOLKSREPUBLIK CHINA
}

\author{
Von OsKar Weggel
}

Vom 13.-17. Januar 1975 tagte in Peking der IV. Nationale Volkskongreß (NVK), an dem 2864 Delegierte teilnahmen und der bis zuletzt völlig geheim geblieben. war. Bezeichnend für den Stellenwert dieses nach der alten Verfassung schon seit 1968 überfälligen Kongresses war die Tatsache, daß kurz vorher, vom 8.-10. Januar, das X. Zentralkomitee (ZK) seine zweite Plenartagung abgehalten hatte, auf der alle wesentlichen Ergebnisse des Kongresses bereits vorweggenommen wurden. Das Plenum hatte die Tagesordnung für den Volkskongreß beschlossen, die Kandidatenlisten für Staatsführung und Regierung zusammengestellt und Teng Hsiaoping zum 6. Stellvertreter Mao Tse-tungs an der Parteispitze ernannt. Die dem NVK vorgelegte Tagesordnung umfaßte drei Punkte, nämlich 1. den Tätigkeitsbericht der Regierung (erstattet durch Chou En-lai), 2. die Wahl sowie die Ernennung der Staatsführung (Bestätigung des alten Ministerpräsidenten, Ernennung von 12 Stellvertretern des Ministerpräsidenten und 49 Ministern) und 3. die Revision der Verfassung.

„Im Namen des ZK der Kommunistischen Partei Chinas" gab Chang Ch'unch'iao, das einzig prominente Mitglied der „Linken“, das unter die 12 Stellvertreter Chou En-lais plaziert worden ist, einen „Bericht über die Revision der Verfassung " ab. Dieser Bericht wurde am 13. Januar verlesen und am 17. Januar angenommen. In der Zwischenzeit wurden also Verfassungstext und Revisionsbericht aller Wahrscheinlichkeit nach vom NVK diskutiert. Am 17. Januar 1975 wurde der „revidierte Text der Verfassung, den das ZK der KPCh dem Ersten Plenum des IV. NVK zur Diskussion vorgelegt hatte, einstimmig angenommen "1. Damit war eine Revisionsarbeit „abgeschlossen“, die, wie Chang Ch'un-ch'iao in seinem Rechenschaftsbericht betonte, „fast fünf Jahre gedauert“ hatte. Der „revidierte Text" brachte nicht allzu viele Neuigkeiten; war doch ein erster Entwurf bereits am 6. September 1970 vom Zweiten Plenum des ZK grundsätzlich gebilligt worden. Dieses 30 Artikel umfassende Dokument war auf dem Umweg über die taiwanesische „Zentralzeitung“ (Chung-yang jih-pao) ins Ausland gelangt und wurde dort u. a. in zwei deutschen Übersetzungen ${ }^{2}$ zugänglich gemacht. Einige Monate später war auch die Präambel verfügbar ${ }^{3}$. Bei der Analyse des „revidierten Textes“ leistet sowohl der Vergleich mit der Verfassung von 1954 (fortan „alte Verfassung“ genannt) wie auch mit dem "Entwurf“ von 1970 hilfreiche Dienste (Der „revidierte Text" wird fortan auch als "neue Verfassung " bezeichnet).

Die neue Verfassung ist - ebenso wie der "Entwurf“ und die "alte Verfassung“ in eine Präambel sowie in vier Abschnitte untergliedert, nämlich I. Allgemeine Grundsätze, II. Staatsorgane, III. Grundrechte und Grundpflichten, IV. Staatssymbole.

\footnotetext{
1 NCNA, 19. 1. 1975.

2 Y. H. Nieh und Oskar Weggel, „Der neue Verfassungsentwurf der Volksrepublik China*; in Verfassung und Recht in UUbersee, Jahrgang 1971, S. $59 \mathrm{ff}$.; Marie-Luise Näth und Werner Pfennig, "Entwurf zur Veränderung der Verfassung der VR China ${ }^{\alpha}$ in: Internationales Asien-Forum, 2. Jahrgang (1971), S. $89 \mathrm{ff}$.

3 Ubersetzung und Kommentar von Dieter Heinzig, „Die Präambel des neuen Verfassungsentwurfs der

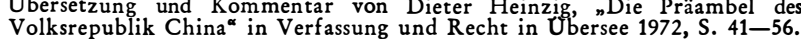


Stand die alte Verfassung noch ganz im Banne des sowjetischen Vorbilds, so ist die nun vorliegende "Revision“ weitgehend von Mao Tse-tungs Vorstellungen geprägt. Den ungleich „politischeren“ Charakter der neuen Konstitution deutet schon das äußere Rahmenwerk an: Hatte das alte Grundgesetz noch 106 Artikel (mit rd. 14000 Wortzeichen) umfaßt, so genügen nunmehr 30 Artikel (mit ungefähr 3400 Zeichen). Hatten die hauptsächlich politische Orientierungslinien enthaltenden „Allgemeinen Grundsätze“ früher nur ein Fünftel des Verfassungstextes eingenommen, so ist es nunmehr ziemlich genau die Hälfte. Rechnet man hier gar noch den Umfang der Präambel hinzu, die ja ebenfalls weitere politische Leitlinien enthält, so machen die "politischen“ Aussagen etwa zwei Drittel des Textes, der juristische Teil nur noch ein Drittel aus. Der Prozeß der „Entpositivierung“ und der Rückkehr des Rechts in die allgemeine Gesellschaftsordnung hat sich damit nicht nur auf dem Gebiet der Gesetzgebung 4 und der Justiz, sondern auch im konstitutionellen Bereich vollzogen.

Nicht nur in der äußeren Aufmachung, sondern auch im inneren Gehalt wurde die Verfassung "maoisiert", wenngleich die starken Formulierungen des „Entwurfs“ von 1970, die ja auf Lin Piao zurückgingen („Die Mao-Tse-tung-Ideen sind lebendig zu lernen und anzuwenden“, „Die politische Arbeit gehört zur Lebenslinie“ etc.) in der Zwischenzeit eliminiert worden sind. Ganz im Stil der von Mao befürworteten "Großen Demokratie" werden aber nach wie vor "große Wandzeitungen" als Vehikel für die Ausführung der sozialistischen Revolution gefordert (Art. 13). Es gilt, die „Revolution anzupacken und die Produktion voranzutreiben" sowie gegen den „Krieg vorbereitet zu sein“ (Art. 10). Die „Landwirtschaft ist als Grundlage und die Industrie als führender Faktor" anzusehen. Die Kader haben sich „mit den Massen eng zu verbinden“, und das Proletariat ist aufgefordert, eine "allseitige Diktatur über die Bourgeoisie im Überbau auszuüben“ (Art. 12). Schon aus diesen wenigen Bestimmungen wird ersichtlich, daß es sich bei dem neuen knappen Text um eine Verfassung der Generalklauseln handelt, deren näherer Gehalt nicht durch „Lückenausfüllung“ oder durch ähnliche juristische Techniken, sondern durch den praktischen politischen Prozeß zu ermitteln ist.

Eine besonders wichtige Entwicklung gegenüber dem „Entwurf“ von 1970 besteht darin, daß jede Form von Personenkult aus dem Text von 1975 verbannt ist. Hierin schließt die neue Verfassung an die alte an. Während nämlich Maos Namen in der Verfassung von 1954 nicht ein einziges Mal aufgetaucht war, wurde er im „Entwurf“ gleich siebenmal genannt. Im "Entwurf“ kehrte Lin Piaos Name dreimal wieder, nämlich einmal in der Präambel, sodann in Artikel 2 und schließlich in Artikel 26. Im Artikel 26 hieß es beispielsweise: „Die fundamentalsten Rechte und Pflichten der Bürger sind folgende: Sie haben den Vorsitzenden Mao Tse-tung und seinen engen Waffenkameraden, den stellvertretenden Vorsitzenden Lin Piao, zu unterstützen ...“. Lins Name ist - verständlich nach seinem Sturz von 1971 und nach der seit 1973 andauernden Anti-Lin-Piao-Kampagne - aus dem Wortlaut der neuen Verfassung verbannt. Aber auch Maos Name taucht nur noch im Zuzusammenhang mit den "Mao-Tse-tung-Ideen “ auf, die in einem Atemzug mit dem Marxismus und dem Leninismus genannt sind (vgl. Absatz 4 der Präambel und Artikel 11/Abs. 1). Eine andere verfassungsrechtliche Kuriosität des „Entwurfes“ ist allerdings auch in dem "revidierten Text“ erhalten geblieben, nämlich die

4 Oskar Weggel, "Die Gesetzgebung in der Volksrepublik China* Verfassung und Recht in Ubersee, 1970, S. 139-166. 
Streichung des Amts des Staatspräsidenten, das der verfemte Liu Shao-ch'i jahrelang bekleidet hatte. Artikel 2 des "Entwurfs" hatte noch vom "Vorsitzenden" Mao gesprochen, ohne allerdings zu präzisieren, ob damit der „Vorsitzende“ des Zentralkomitees oder aber der „Vorsitzende“ des Staates gemeint war. Das Fehlen der "Vorsitzenden"-Formel in der neuen Verfassung macht nun klar, daß Mao schon damals in seiner Eigenschaft als Erster Mann der Partei angesprochen war!

Im folgenden sollen die fünf Abschnitte der Verfassung im einzelnen betrachtet werden.

\section{Die Präambel}

Die „Präambel“ (hsü-yen) der neuen Verfassung ist nach demselben formalen Schema aufgebaut wie die der alten Verfassung und die des „Entwurfes“. Sie gliedert sich in zwei große Teile, die sich als "Rückblick“ und „Ausblick“ bezeichnen ließen und die sich ihrerseits wieder zweifach untergliedern: Der „Rückblick“ ist zunächst auf die Machteroberung bis 1949 und sodann auf die „letzten 20 Jahre und mehr" gerichtet, während der "Ausblick“ zunächst die Innenpolitik und dann die Außenpolitik beleuchtet ${ }^{5}$. Der Sieg des chinesischen Volkes über „Imperialismus, Feudalismus und bürokratischen Kapitalismus" wird in der neuen Verfassung nicht mehr, wie im „Entwurf“, dem Großen Führer, Vorsitzenden Mao Tse-tung und der Kommunistischen Partei Chinas zugeschrieben, sondern nurmehr dem „Volk unter der Führung der KP“. Dieser Erfolg wird auch heute noch als ein "Sieg der Neuen Demokratie“ bezeichnet - ein Ausdruck, der im "Entwurf“ gestrichen war! Was die Zeit seit 1949 anbelangt, so haben sich hier Änderungen ergeben, die in der neuen Verfassung ihren Niederschlag finden mußten. Hatte es die alte Verfassung mit einer Gesellschaft der „Übergangsperiode“ zu tun, so liegen nunmehr seit fast 20 Jahren (genauer: seit 1956) sozialistische Verhältnisse in der VR China vor - ein Tatbestand, der in der neuen Verfassung - wie übrigens auch schon im „Entwurf“ ausführlich Berücksichtigung gefunden hat. Wie Chang Ch'un-ch'iao in seinem Rechenschaftsbericht deutlich macht, besteht zwischen der alten und der neuen Verfassung kein Bruch, sondern ein kontinuierlicher Übergang. Als Verfassung, die nicht nur allgemeine Regeln, sondern auch fundamentale Leitlinien enthalte, habe die Konstitution von 1954 - „Chinas erste Verfassung vom sozialistischen Typ“ - ihren Zweck voll erfüllt. In ihrer Eigenschaft als fundamentale Leitlinie habe sie die historischen Erfahrungen zusammengefaßt, die Siege des Volkes konsolidiert und klare Ziele gesteckt. Die Praxis der vergangenen Jahrzehnte habe bewiesen, daß die alte Verfassung "richtig“ war. Ihre Grundprinzipien seien deshalb auch heute noch anwendbar. Jedoch müßten auch einige grundlegende Änderungen berücksichtigt werden.

Der zweite Teil der Präambel, der sich mit den künftigen innen- und außenpolitischen Leitlinien beschäftigt, beginnt mit einer Darlegung der revolutionären Generallinie, wie sie von Mao Tse-tung bereits 1958 erstmals definiert wurde: nämlich der Theorie der "permanenten Revolution“. Es ist bemerkenswert, daß die beiden

\footnotetext{
5 Eine formell etwas andere Einteilung benutzt Heinzig a. a. O., der von vier Themenkomplexen spricht:

a) historischer Teil, der bis zur kommunistischen Machtübernahme führt

b) von 1949 bis zur Gegenwart

c) gegenwärtige innenpolitische Situation

d) Prinzipien der AuBenpolitik.
} 
gegensätzlichen Begriffspaare „permanente Revolution“ (Pu-tuan ke-ming) und „fortgesetzte Revolution“ (Chi-hsü ke-ming), die noch im "Entwurf“ har nebeneinander gestanden und Interpretationsschwierigkeiten bereitet hatten ${ }^{6}$, in der "neuen Verfassung" nurmehr in Form des "Chi-hsü ke-ming" vertreten sind. Die Vermutung Stuart R. Schrams, daß die divergierenden Formulierungen mit Auseinandersetzungen um Lin Piao zusammenhingen, hat sich also nachträglich bestätigt. Auch hier wurden also Lin Piao-Reste eliminiert und die Verfassung ganz auf das „Mao-Tse-tung-Denken“ zugeschnitten ${ }^{6 a}$. Im Gegensatz zu der Chruschtschow'schen These von einer "Partei des ganzen Volkes“ und von einem "Staat des ganzen Volkes" wird in der sozialistischen Gesellschaft Chinas, die ja nach wie vor durch Klassen, Klassenwidersprüche und Klassenkämpfe geprägt ist, nur eine „Partei der Diktatur des Proletariats" und ein "Staat der Diktatur des Proletariats“ anerkannt. Die alte Formulierung von einer „breiten volksdemokratischen Einheitsfront aller demokratischen Klassen, Parteien, Gruppen und Massenorganisationen unter Führung der KP Chinas" ist m. a. W. durch eine Ein-Klassen-Front ersetzt worden. Wie Chang Ch'un-ch'iao betont, ist die Erkenntnis von der Unausweichlichkeit der "permanenten Revolution unter der Diktatur des Proletariats“ die „Haupterkenntnis, die wir gewonnen haben, und auch der Grundgedanke bei der Revision der Verfassung“. Bezeichnend aber ist immerhin, daß der „Sozialimperialismus" (also die Sowjetunion!) nicht als eine der Hauptbeweggründe für die Unausweichlichkeit weiterer Klassenkämpfe herausgestellt wird (so noch im „Entwurf“!). Im „Entwurf“ waren im Zusammenhang mit der Innenpolitik noch die fünf Säulen aufgeführt, auf denen die damalige VR China beruhte (Mao Tse-tung, die Partei, Mao-Tse-tung-Ideen als theoretische Grundlage, das Volk und die Armee). Dieser Hinweis ist in der neuen Verfassung entfallen, der insofern der alten Verfassung wesentlich ähnlicher ist als dem „Entwurf“. „Entwurf“ und „neue Verfassung" kommen sich aber insofern wieder näher, als die nachdrückliche Betonung der Minoritäten-Besonderheiten (Abs. 5 der Präambel von 1954) hier unterblieben ist. Die neue Verfassung bringt am Ende ihres innenpolitischen Ausblicks schließlich noch eine Reihe von Parolen, wie sie weder in der alten Verfassung noch im „Entwurf“ aufgetaucht waren. Sie lauten: „Wir sollen korrekt zwischen Widersprüchen im Volk und Widersprüchen zwischen uns und unseren Feinden unterscheiden und sie richtig behandeln." „Wir sollten die drei großen revolutionären Bewegungen des Klassenkampfes, des Produktionskampfes und des wissenschaftlichen Experiments vorantreiben"; weiterhin ergeht die Aufforderung, den "Sozialismus unabhängig und gestützt auf die eigene $\mathrm{Kraft}^{\text {" }}$ aufzubauen, wobei die alte Generallinie von 1958, die auf „noch größere, schnellere, bessere und zusätzliche ökonomische Ergebnisse" hinauslief, wiederholt wird. Schließlich taucht die seit den Ussuri-Auseinandersetzungen vielleicht wichtigste Parole auf: „Vorbereitung auf den Verteidigungsfall und auf Naturkatastrophen; alles für das Volk!“. Es fällt auf, daß die meisten dieser Parolen „produktionsorientiert" sind. Hierdurch unterscheiden sie sich von den eher "politisch“ eingefärbten Aussagen der „LinPiao-Präambel“ von 1970.

Im außenpolitischen „Ausblick“ schließlich kehrt die Dreierformel wieder, wie sie sich seit den sinosow jetischen Ideologieauseinandersetzungen in den Jahren 1963/64

6 Ausführlich zu dieser Problematik Heinzig, a. a. O., S. 46-49, mit ausführlichen Literaturnachweisen.

6a) Beide Begriffe sind im Deutschen nicht ganz adäquat wiederzugeben. Der. Begriff "Chi-hsü ke ming“ wird Mao zugeordnet. Im folgenden wird allerdings der in Deutschland eingeführte Begriff der "permanenten Revolution" weiterverwendet. 
im chinesischen Sprachschatz fest verankert haben: Gegenüber den sozialistischen Ländern ist "proletarischer Internationalismus“, gegenüber den um ihre Emanzipation kämpfenden Völkern der Dritten Welt "materiell wirksame Unterstützung“ und gegenüber Ländern mit anderer Gesellschaftsordnung "friedliche Koexistenz" nach den bekannten Fünf Prinzipien zu üben. Erstaunlicherweise ist die erstmals von Teng Hsiao-ping auf der Welt-Rohstoffkonferenz vorgetragene neue „DreiWelten-Theorie“ in dem Verfassungstext formell unerwähnt geblieben. Teng hatte damals betont: „Durch das Auftreten des Sozialimperialismus hat das nach dem Zweiten Weltkrieg eine Zeitlang existierende Sozialistische Lager zu bestehen aufgehört. Infolge des Gesetzes der ungleichmäßigen Entwicklung des Kapitalismus gerät auch der imperialistische Block im Westen in Auflösung. Sieht man sich die Anderung der internationalen Beziehungen an, so gibt es heute in der Welt drei Teile, drei Welten, die sowohl voneinander abhängig sind als auch in Widerspruch zueinander stehen. Die USA und die Sowjetunion bilden die Erste Welt. Die Entwicklungsländer in Asien, Afrika und Lateinamerika sowie in anderen Gebieten bilden die Dritte Welt. Und die entwickelten Länder, die sich zwischen diesen beiden Welten befinden, bilden die Zweite Welt"7. Diese „Drei-Welten-Formel“ hatte die alte $Z$ wischenzonentheorie von 1964 zumindest formell abgelöst ${ }^{8}$. Betrachtet man den Wortlaut des vorletzten Präambelabsatzes, so wird man allerdings entdecken, daß die „Drei-Welten-Formel“ zumindest impliciter mit berücksichtigt ist. Dies geht aus dem ganzen dortigen Gedankengang hervor, nicht zuletzt aber aus der Bemerkung, daß China „niemals eine Supermacht" werden solle und daß es dem chinesischen Volk darum zu tun sein müsse, „der imperialistischen und sozio-imperialistischen Aggressionspolitik sowie dem Hegemonismus der Supermächte Widerstand zu leisten". Teng Hsiao-ping hatte ja seinerzeit die Natur der beiden „Supermächte“ näher definiert: „Was ist eine Supermacht? Sie ist ein imperialistisches Land, das überall mit Aggression, Intervention, Kontrolle, Subversion und Ausplünderung gegen andere Länder vorgeht und die Vorherrschaft über die Welt anstrebt. Wird in einem großen sozialistischen Land der Kapitalismus restauriert, wird er sich unvermeidlich in eine Supermacht verwandeln. Die Große Proletarische Kulturrevolution der vergangenen Jahre und die Bewegung zur Kritik an Lin Piao und Konfuzius, die zur Zeit ganz China erfaßt, sind darauf gerichtet, die Restauration des Kapitalismus zu verhindern, das sozialistische China davor zu bewahren, daß es die Farbe wechselt, und sicherzustellen, daß China immer auf der Seite der unterdrückten Völker und Nationen steht. Sollte China eines Tages die Farbe wechseln, sich in eine Supermacht verwandeln und ebenfalls in der ganzen Welt wie ein Tyrann herumkommandieren, überall die anderen demütigen, angreifen und ausbeuten, dann müssen die Völker der Welt diesem China das Schild des Sozialimperialismus umhängen, es entlarven, bekämpfen, und es mit dem chinesischen Volk gemeinsam niederschlagen "9. So gesehen fügt sich die Teng-Rede gut in den neuen Präambel-Text ein. Teng Hsiao-ping, der während der Kulturrevolution als Revisionist angeklagte Pragmatiker, hat damit indirekt eine Bestätigung seiner Politik und seiner außenpolitischen Handschrift erfahren. Bemerkenswert sowohl für den „Entwurf“ wie für die neue Verfassung, die im

\footnotetext{
7 PRu 1974, Nr. 15, S. 8.

8 Näheres dazu Oskar Weggel, „Zur Armut in der Dritten Welt: Ursache und Therapie (China bei der UN-Rohstoffkonferenz)“, "CHINA aktuell ${ }^{\alpha}$, Mai 1974, S. 228-236 (hier 233 ff.).

9 Peking Rundschau 1974, Nr. 15, S. 13
} 
außenpolitischen Teil weitgehend übereinstimmen, ist der Aufbau eines „Feindbildes"10, wie es für die ganz im Geiste von Bandung stehende, weitaus „versöhnlichere“ Verfassung von 1954 noch indiskutabel war. Damals auch war die „unverbrüchliche Freundschaft zur Großen Union der Sozialistischen Sowjetrepubliken und zu den Ländern der Volksdemokratie" beschworen worden!“

Während im sozialistischen China selbst das "Proletariat" aufgerufen ist, die Klassenfeinde zu unterdrücken, appelliert China im internationalen Bereich nicht nur an die wenigen, noch verbliebenen sozialistischen Länder (Nordkorea, Nordvietnam, Rumänien und Albanien), sondern auch an die Länder der Zweiten und Dritten Welt, eine möglichst breitangelegte „Front“ gegen die beiden Supermächte zu bilden. Die klassenkämpferische Tendenz, von der die neue Verfassung auch sonst geprägt ist, taucht also auch hier - im außenpolitischen Bereich - in modifizierter Form auf.

Vergleicht man zusammenfassend die neue Verfassung mit dem „Entwurf“ von 1970, so fällt die weitgehende „Entpersonalisierung“ sowie die „Produktionsorientiertheit" der Parolen auf. Zieht man andererseits Parallelen zwischen der neuen und der alten Verfassung von 1954, so ergeben sich als wichtigste Neuerungen das Bekenntnis zur permanenten Revolution im Innern und zum Aufbau eines „Feindbildes“ im äußeren Bereich.

\section{2. „Allgemeine Grundsätze“}

Mit immerhin 15 Artikeln schneiden sowohl der "Entwurf“ wie auch die neue Verfassung gegenüber den 20 Artikeln der alten Verfassung quantitativ nicht schlecht ab. Am klarsten treten die Entwicklungen zwischen 1954 und 1975 in den Artikeln 1 und 2 zutage. 1954 heißt es: „Die VR China ist ein volksdemokratischer Staat, der von der Arbeiterklasse geführt wird und seine Grundlage in dem Bündnis der Arbeiter und Bauern hat“. 1975: „Die VR China ist ein sozialistischer Staat der Diktatur des Proletariats, der von der Arbeiterklasse geführt wird und seine Grundlage in dem Bündnis der Arbeiter und Bauern hat". Ebenso unmißverständlich sind die Unterschiede in Artikel 2: 1954 heißt es: „Alle Macht in der VR China gehört dem Volk. Die Organe, durch die das Volk die Macht ausübt, sind der Nationale Volkskongreß und die örtlichen Volkskongresse". Der Text von 1975: „Die Kommunistische Partei China ist der führende Kern des ganzen chinesischen Volkes. Die Arbeiterklasse übt ihre Führung über den Staat durch ihre Vorhut, die KPCh, aus. Der Marxismus-Leninismus und die MaoTse-tung-Ideen sind die theoretische Basis, die das Denken unserer Nation anleiten." (Dann erst wird - in Art. 3 - die Formulierung des Art. 2 der alten Verfassung wiederholt!) An dieser Stelle tritt die Führungsrolle der Partei, die ja 1954 angesichts des damaligen Vier-Klassen-Bündnisses im Zeichen der Neuen Demokratie noch nicht so unverhüllt hervortreten konnte, klar zutage. Artikel 2 des „Entwurfs“ hatte demgegenüber einen stark personenbezogenen Akzent: „Der Vorsitzende Mao Tse-tung ist der Große Führer aller Nationalitäten im ganzen Staat, er ist Oberhaupt unseres Staates der Diktatur des Proletariats, er ist Oberbefehlshaber der gesamten Nation und aller Streitkräfte. Stellvertretender Vor-

10 vgl. dazu Heinzig, a. a. O., S. $50 \mathrm{ff}$. 
sitzender Lin ist der enge Waffenfreund und Nachfolger des Vorsitzenden Mao, er ist stellvertretender Oberbefehishaber des ganzen Staates und aller Streitkräfte. Mao Tse-tungs Denken ist die Richtlinie für alle Arbeit des Volkes der ganzen Nation“. Sodann (Artikel 3): „Alle Macht in der VR China gehört dem Volk ...". Ubereinstimmung besteht bei allen drei Texten also darin, daß „alle Macht dem Volk gehört" und daß die Organe, durch die das Volk seine Macht ausübt, die Nationalen Volkskongresse der verschiedenen Ebenen sind. Gemeinsam ist ihnen "das Prinzip des Demokratischen Zentralismus“.

Während aber die Mitglieder der Kongresse nach der alten Verfassung noch „gewählt" werden mußten (vgl. dort z. B. Art. 23), werden sie sowohl nach dem „Entwurf“ als auch nach der neuen Verfassung „durch demokratische Konsultation" ernannt (Art. 3 beider Texte).

Nach den Bestimmungen über Führung und Führungsprinzipien folgt der zweite Abschnitt über die Minoritätenfrage. Die neue Verfassung hat hier den Wortlaut des „Entwurfs“ (Art. 4) übernommen. Gegenüber der alten Verfassung sind keine substantiellen formalen Änderungen zu bemerken. Nach wie vor gilt die VR China als ein aus vielen Nationalitäten bestehender Einheitsstaat, in dem alle Minoritäten gleichberechtigt sind, in dem die nationalen Minderheiten ferner ihre eigene Sprache und ihre eigene Schrift anwenden können, wobei allerdings hinzugefügt wird, daß Gebiete mit nationaler Autonomie unabtrennbare Bestandteile der Volksrepublik sind. Seit dem Ende der Lin-Piao-Ära hat sich auf dem Gebiet der Minoritätenpolitik eine Wandlung vollzogen, die den Minoritäten in der Tat wieder mehr Autonomie einräumt. Einige türkische und kasachische Völker konnten beispielsweise in der Zwischenzeit zur Benutzung der lateinischen Schrift übergehen. Dies ist um so bemerkenswerter, als während der Kulturrevolution Minoritätenfragen eine Zeitlang nur noch als Klassenkampfprobleme behandelt worden waren.

Ein dritter Abschnitt im Rahmen der Allgemeinen Grundsätze ist den Produktionsverhältnissen gewidmet. Die alte Verfassung hatte noch vier Hauptformen des Eigentums anerkannt, nämlich Staatseigentum (Volkseigentum), genossenschaftliches (kollektives) Eigentum der Werktätigen; Eigentum der individuell produzierenden Werktätigen und Eigentum der Kapitalisten. Die neue Verfassung kennt nur noch zwei Eigentumsformen: Volkseigentum und Kollektiveigentum (Artikel 5). Weggefallen sind alle früheren Bestimmungen, die der sogenannten Bourgeoisie Existenz, Vermögen und Erbrecht garantierten. Bemerkenswert bei der neuen Regelung, die im übrigen wortwörtlich mit dem „Entwurf“ übereinstimmt, ist jedoch die Tatsache, daß es Arbeitern erlaubt ist, „einer individuellen Tätigkeit nachzugehen, vorausgesetzt, daß sie sich im Rahmen der Gesetze hält und daß andere nicht ausgebeutet werden". Damit ist dem individuellen Erwerbsstreben prinzipiell ein Türspalt geöffnet worden. Immerhin heißt es dann - und hier scheint den Linken ein Zugeständnis gemacht worden zu sein - im nächsten Satz: „Gleichzeitig sollen sie (diese Arbeiter!) dazu angeleitet werden, nach und nach den Weg der sozialistischen Kollektivierung zu beschreiten“. Im Gegensatz zu 1954, wo Grund und Boden noch Individualeigentum sein konnte (Artikel 11, 13), ist an solchen „Sachen“ heute nur noch sozialistisches Eigentum möglich.

Neu gegenüber 1954 sind auch die Bestimmungen über die „ländlichen Volkskommunen“ (Artikel 7), die ja erst vier Jahre nach der Verkündung der damaligen Verfassung zum Entstehen kamen. Zu bedenken ist allerdings, daß die Regelung, 
wie sie heute in Artikel 7 getroffen wird, nur noch wenige Züge mit der 1958 konzipierten modellhaften "Sputnik-Kommune" gemein hat ${ }^{11}$. Auch damals war zwar bereits von Produktionsbrigaden und Produktionsmannschaften die Rede. Doch welch ein Unterschied in den Rechten und Befugnissen der Volkskommune:

- Was zunächst das Eigentum an landwirtschaftlichen Produktionsmitteln anbelangt, so stand es damals uneingeschränkt der Volkskommune zu, während es nach der heutigen Verfassung auf „drei Stufen“ aufgeteilt ist, wobei die Produktionsmannschaft sogar als subsidiärer Eigentümer gilt.

- Auch in den Produktionszuständigkeiten lag das Schwergewicht damals bei der Volkskommune: Sie stellte die jährlichen Produktionspläne auf und leitete auch die landwirtschaftliche und industrielle Produktion sowie die Handelsorganisation. Außerdem war sie für den Milizaufbau zuständig. Die Produktionsbrigaden waren lediglich Grundeinheiten für die Organisation der Arbeit und verwalteten die kleineren Fabrikbetriebe. Den Produktionsmannschaften gar stand nur die Verwaltung an kleinen Maschinen zu. Im Gegensatz dazu stellen Produktionsbrigade und Produktionsmannschaft heute ihre eigenen Pläne auf (Vor der Kulturrevolution konnten sie sich im Wege von „Fixierungen" und "Garantien" sogar ein erhebliches Maß an Freiheiten und Entlohnungsansprüchen gegenüber den Volkskommunen verschaffen, ein Verhalten, das während der Kulturrevolution als „Ökonomismus“ verurteilt wurde!). Vor allem aber sind Produktionsbrigaden und Produktionsmannschaften verantwortlich für die von ihnen errichteten lokalen Industriebetriebe - eine Aussage, die im Verfassungstext zwar nicht ausdrücklich zutage tritt, die aber durch die herrschende Praxis anerkannt ist.

- Was die Distributionszuständigkeiten anbelangt, so war bei der Sputnik-Kommune von 1958 die Produktionsbrigade lediglich für Durchführungskontrolle und für die technische Rechnungsführung verantwortlich, während Gewinn und Verlust direkt auf das Konto der Volkskommune gingen. Heute ist die Produktionsmannschaft "Grundverrechnungseinheit" und führt nicht nur ihr eigenes Arbeitspunkte-Konto, sondern trägt auch Gewinn und Verlust in eigener Verantwortung (Art. 7, Abs. 2 der neuen Verfassung) ${ }^{\mathbf{1 2}}$.

- Bemerkenswert ist auch die Bestimmung in Artikel 7/Abs. 3, daß nämlich die einzelnen Bauern Privatparzellen zugeteilt bekommen (Art. 7/Abs. 3). Obwohl das Privateigentum an Land, Vieh und Geräten abgeschafft wurde, steht den Kommunenmitgliedern also heute wieder privates Land und eine kleine private Viehzucht zur Verfügung. Nicht selten werden ihnen pro Kopf 7 Prozent eines $\mathrm{Mu}(15 \mathrm{Mu}=1 \mathrm{ha})$ zugebilligt. Die auf solchen Parzellen erzeugten Produkte können auf partiell freien Märkten weiterveräußert werden ${ }^{13}$.

In Artikel 10 haben sich zwischen „Entwurf“ und neuer Verfassung wiederum Unterschiede ergeben, die zurückzuführen sind auf den Einfluß Lin Piaos. Im Entwurf hieß es: „Politische Arbeit ist die Lebenslinie aller Arbeit. Der Staat sorgt dafür, daß die Revolution angepackt und die Produktion vorangetrieben wird." Statt dessen heißt es heute sofort: „Der Staat wendet das Prinzip an, daß die Revolution angepackt und die Produktion vorangetrieben wird ..."Neu hinzugekom-

11 Satzung der Sputnik-Volkskommune in JMJP (= Pekinger Volkszeitung) vom 7. 9. 1958

12 Oskar Weggel, „Wie eine Volkskommune im Jahre 1974 funktioniert " „CHINA aktuell ${ }^{\alpha}$ März 1974, S. $122-135$ (134 f.) 13 Ebd., S. 131 . 
men ist auch der auf Mao Tse-tung zurückgehende Satz, daß die „Landwirtschaft als Grundlage und die Industrie als führender Faktor" zu gelten habe. Schließlich wird abweichend vom „Entwurf“ darauf hingewiesen, daß sowohl die zentralen wie auch die lokalen Behörden ihre Initiative voll entfalten sollen. Offensichtlich wird damit der Dezentralisierung wieder stärker das Wort geredet. Auch die Bestimmungen über die „Arbeit“ haben sich im Laufe der Jahre verändert: 1954 hieß es (Art. 16): „Die Arbeit ist für alle arbeitsfähigen Bürger der VR China eine Sache der Ehre. Der Staat fördert die Aktivität und das Schöpfertum der Bürger in ihrer Arbeit.“ Der Wortlaut von 1975 (Art. 9): „Der Staat geht von folgenden sozialistischen Prinzipien aus: ,Wer nicht arbeitet, soll auch nicht essen' und ,Jeder nach seinen Fähigkeiten, jedem nach seiner Leistung'“ Damals wie heute kommt dem Staat unter anderem die Aufgabe zu, das Arbeitseinkommen, die Ersparnisse, die Wohnung und das Recht auf andere Mittel der Lebenshaltung der Bürger zu schützen.

In einem vierten Abschnitt innerhalb der „Allgemeinen Grundsätze“ (Art. 11) werden die Verwaltungskader angesprochen. Noch 1954 hieß es (Art. 18): „Alle Mitarbeiter der staatlichen Organe müssen der volksdemokratischen Ordnung treu ergeben sein, sich der Verfassung und den Gesetzen unterordnen und mit all ihren Kräften dem Volk dienen." Wesentlich „klassenbetonter" heißt es in der neuen Verfassung (Art. 11): „Alle Mitarbeiter der staatlichen Organe müssen ernsthaft den Marxismus-Leninismus und die Mao-Tse-tung-Ideen studieren, der proletarischen Politik das Kommando einräumen, die Bürokratie bekämpfen, enge Bindungen zu den Massen unterhalten und ernsthaft dem Volk dienen. Kader aller Ebenen müssen an kollektiver Produktionsarbeit teilnehmen." Außerdem wird bestimmt, daß sich Staatsorganisationen nach dem Grundsatz der "Drei-in-eins-Kombination“ (alt, mittel und jung) etablieren sollen. An dieser Stelle fällt der Unterschied zum „Entwurf“ von 1970 besonders ins Auge: Im damaligen Artikel 11 waren typische Lin-Piao-Formulierungen verwendet worden, die inzwischen eliminiert werden mußten, so z. B. die Bestimmung, daß „alle Mitarbeiter in den Staatsorganen die Mao-Tes-tung-Ideen lebendig zu studieren und lebendig anzuwenden haben . ..., daß sie besonderen Wert auf proletarische Politik legen müßten . . . und daß die „Führungsorgane die ,revolutionäre Dreierallianz zwischen Armeevertretern (!), Funktionären und Massen sowie zwischen alt, mittel und jung' herbeizuführen haben“. Die alte Dreierformel, die auf eine Vereinigung von Militärs, Kadern und Massenrepräsentanten abzielte - und zwar insbesondere im Zusammenhang mit dem Aufbau der Revolutionskomitees! — ist seit 1971 ganz von der „Alt-mitteljung “-Formel verdrängt worden.

In einem fünften Abschnitt (Artikel 12-14) schließen sich klassenkämpferische Parolen an, wie sie der alten Verfassung noch unbekannt waren. Artikel 12, demzufolge das Proletariat im Überbau über die Bourgeoisie eine umfassende Diktatur auszuüben hat, entstammt dem Vokabular der Kulturrevolution. Dasselbe gilt von Artikel 13, in dem "große Wandzeitungen" als Ausdruck der sozialistischen Demokratie gefordert werden. Die Formulierung „Großes Singen und Blühen, Große Debatten . ..., wie sie noch in Artikel 13 des „Entwurfs" enthalten sind, tauchen in der neuen Verfassung nur noch in „abgekühlter“ Form auf („freie und offene Rede“ etc., Art. 13). Artikel 14 baut - parallel zur Präambel - ein „Feindbild“ auf, wie es in der Verfassung von 1954 noch nicht enthalten war. Danach „unterdrückt der Staat alle verräterischen und konterrevolutionären Aktivitäten . . . und 
sorgt dafür, daß nach Maßgabe des Gesetzes Grundherren, Reiche Bauern, reaktionäre Kapitalisten, Konterrevolutionäre u. a. unerwünschte Elemente ihre politischen Rechte ... verlieren“. Artikel 15 schließlich legt die Stellung der Volksbefreiungsarmee und der Volksmiliz im Gesellschaftssystem fest. Der Wortlaut ist identisch mit dem des "Entwurfs", wobei allerdings in der Zwischenzeit noch ein weiterer Satz hinzugekommen ist, der folgendermaßen lautet: „Der Vorsitzende des ZK der KPCh kommandiert die bewaffneten Streitkräfte des Landes." Mao Tse-tung ist damit konstitutionell zum Oberbefehlshaber der Armee ernannt. Schon nach Artikel 42 der Verfassung von 1954 lag der Oberbefehl über die Streitkräfte des Landes beim „Vorsitzenden der VR China“, also dem Staatspräsidenten. Da dieses Amt inzwischen entfallen ist, mußte eine neue Lösung gefunden werden. Die noch stärkere Einbeziehung des Parteiapparats in den Staatsapparat wird damit auch konstitutionell gebilligt. Noch 1954 (Art. 20) war die Armee damit beauftragt, "die Errungenschaften der Volksrevolution und die Erfolge des Staates beim nationalen Aufbau sowie die Souveränität, territoriale Integrität und Sicherheit des Landes zu schützen". Dieser Aufgabenkatalog ist jetzt erweitert. Die Streitkräfte schützen nunmehr „die Errungenschaften der sozialistischen Revolution und des sozialistischen Aufbaus, verteidigen die Souveränität, territoriale Integrität und Sicherheit des Staates und kämpfen gegen Subversion und Aggression von seiten des Imperialismus, Sozio-Imperialismus (!) und ihre Lakaien". Auch findet sich das inzwischen schon klassisch gewordene Prinzip, daß die Streitkräfte nicht nur zu kämpfen, sondern auch zu produzieren und zu arbeiten haben, nunmehr expressis verbis im Verfassungstext. Die alte Formulierung allerdings, daß die Armee auch "Massenarbeit“ (d. h. politische Erziehung unter der Bevölkerung!) zu leisten und als „Modell für das ganze Volk“ zu gelten hat, ist mit Rücksicht auf die negativen Erfahrungen mit den Streitkräften während der Kulturrevolution im Verfassungstext nicht aufgenommen worden.

\section{Die Staatsorgane}

Wie ernst es der Pekinger Führung gerade mit der Revolution der Staatsapparatur ist, geht vor allem aus der radikalen Kürzung des Abschnittes II hervor, in dem die „Staatsorgane" abgehandelt sind. Wo in der alten Verfassung 64 Artikel notwendig waren, genügen heute 10 (Artikel 16-25). Anstelle der sechs alten Organe (Nationaler Volkskongreß, Staatspräsident, Staatsrat, örtliche Volksversammlungen und Volksausschüsse, Selbstverwaltungskörperschaften, Volksgerichte sowie Volksanwaltschaft) existieren - nach Abschaffung des Präsidentenamtes - nur noch fünf und auch diese nur noch in stark veränderter Form.

a) Der Nationale Volkskongreß hat seine Stellung als „Oberstes Organ der Staatsgewalt" sowie seine wichtigsten Rechte (Verfassungsänderung, Gesetzeserlaß, Haushalt, Bestellung des Staatsrats usw.) im wesentlichen behalten. 1975 wurde er von insgesamt 2885 Deputierten beschickt (vgl. 1954-1957: 1226, 1959-1964: 1226, 1964: 3040). Die Delegierten wurden, wie es heißt, „nach umfangreichen demokratischen Beratungen und wiederholten Diskussionen" gewählt. Aus den offiziellen Protokollen läßt sich nicht entnehmen, ob ein ähnlicher Schlüssel wie für die 3040 Abgeordneten von 1964 (d. h. 1 Delegierter auf 400000 Einwohner in den Provinzen oder aber auf 50000 Einwohner in den Großstädten) angewendet wurde 
und wer die neuen Delegierten vorgeschlagen und gewählt hat. Von den neugewählten Abgeordneten sind 72 Prozent Arbeiter, Bauern und Soldaten und 22 Prozent Frauen. Unter ihnen befinden sich auch Vertreter der Minderheiten und nicht weniger als 12 Abgeordnete für Taiwan!

Seine Stellung hat im wesentlichen auch der Ständige Ausschuß des NVK beibehalten, der bisher faktisch das machtvollste Staatsorgan war; zusätzlich wurde seinem Vorsitzenden nun noch das Recht auf Akkreditierung auswärtiger Gesandter übertragen (Seit dem Sturz des früheren Staatspräsidenten hatte Tung Pi-wu als „Präsident in Vertretung" dieses Recht ausgeübt. Die Änderung wurde den ausländischen Botschaften Ende Januar 1975 per Zirkular des Pekinger Außenministeriums bekanntgemacht). Der Ständige Ausschuß setzt sich zusammen aus dem Vorsitzenden, 22 Stellvertretern (darunter 3 Frauen) und 144 Mitgliedern (darunter 39 Frauen).

Bemerkenswert ist die Verlängerung der Tagungsdauer des NVK. 1954 war hier eine Vierjahresfrist vorgeschrieben, die jedoch zuletzt um nicht weniger als 7 Jahre überschritten wurde (d. h. der IV. Nationale Volkskongreß hätte eigentlich schon 1968 tagen müssen!). Nach Art. 16/Abs. 3 der neuen Verfassung wird der NVK jetzt auf 5 Jahre gewählt. Bei „Vorliegen besonderer Umstände“ kann diese Frist jedoch auch verlängert werden. Nach Art. 16/Abs. 4 hat der NVK jedes Jahr eine Sitzung abzuhalten, die allerdings auch vorgezogen oder aber verschoben werden kann! Für den Fall neuer personeller Zwistigkeiten, die ja regelmäßig der Anlaß für die Nichteinberufung des NVK sind (Fraktionsstreitigkeiten zwischen Liu Shao-ch'i und Mao bzw. später zwischen Lin Piao und Mao!) hat man sich also eine Hintertür freigehalten.

b) Im wesentlichen unverändert blieb auch Chou En-lais Staatsrat, der zwar ziemlich farblos definiert wird, nach wie vor aber die "Zentrale Volksregierung" verkörpert. Der neue Staatsrat, die Regierung der Volksrepublik, besteht aus 1 Ministerpräsidenten (Chou En-lai wurde in seinem Amt bestätigt.), 12 Vizepremiers und 29 Ministern. Von den 29 Ministern wurden beim IV. NVK 17 neu ernannt. 3 der 29 Minister sind eigentlich Kommissionsvorsitzende (nämlich der Staatlichen Planungskommission, der Staatlichen Kommission für Investbau und der Staatlichen Kommission für Körperkultur und Sport). Sie nennen sich aber nunmehr „Minister in Wahrnehmung der ... -Kommission“. Im Vergleich zu 1964, als 16 Stellvertretende Ministerpräsidenten und 46 Minister amtiert hatten, ist die Regierung stark vereinfacht worden - eine Folge der bürokratiefeindlichen Kulturrevolution. Von den Fachministern gehören 3 zugleich dem Gremium der stellvertretenden Ministerpräsidenten an, nämlich die Chefs der Planungskommission sowie der Investbau-Kommission und der Minister für Öffentliche Sicherheit. Bei den 12 Stellvertretern wurde offensichtiich auf Balance geachtet: $\mathrm{Zu}$ ihnen gehört ein exponierter "Linker" (Chang Ch'un-ch'iao, dem auch - Regie Chou En-lais! das Privileg der Verlesung des Verfassungsberichts erteilt wurde), ferner Arbeiterund Bauern-„Modelle“, ein Militär, mehrere Wirtschaftsfachleute und - erstaunlich schwach vertreten - nur 1 Frau. Neu gegenüber der Verfassung von 1954 ist die Bestimmung, daß der Staatsrat den nationalen Wirtschaftsplan sowie den Haushaltsplan „formuliert und ausführt“ (Art. 20). Früher durfte er nur „Maßnahmen zur Realisierung der Wirtschaftspläne und zur Durchführung des Staatshaushalts treffen" (Art. 49, 7, der Verfassung von 1954). Damals entschied nämlich der NVK über die Volkswirtschaftspläne und prüfte den Staatshaushalt. Aus der Novellie- 
rung wird allerdings nicht recht klar, wie weit sich die Planungs- und Haushaltsbefugnisse des Staatsrates wirklich geändert haben.

c) Auf der Ebene der lokalen Organe zeigt es sich am deutlichsten, welchen Einfluß die Kulturrevolution auf die Organstruktur ausgeübt hat. Gerade dort waren ja die alten Partei- und Volksausschüsse, die sich noch nach der Verfassung von 1954 etabliert hatten, in den Wirren der Jahre 1966 - 1968 weitgehend zerstört worden. Die Regionalorgane waren in der alten Verfassung noch durch 14 ausführliche Artikel geregelt (Art. 53-66). Die neue Verfassung bringt hier nur noch drei kurze, wenig ins Detail gehende Bestimmungen (Art. 21-23). Im Regionalbereich sollen in Zukunft die Revolutionskomitees als ständige regionale Regierungen fungieren und als solche den örtlichen Volkskongressen verantwortlich sein. Wirkliche Befehlsgewalt dürften freilich auch in Zukunft nur die lokalen Parteiausschüsse ausüben. Was die Revolutionskomitees von den bisherigen lokalen Volksausschüssen unterscheidet, ist vor allem ihre Mission als "revolutionierte“ Organe, in denen der Bürokratismus keinen Halt mehr finden soll. Regelmäßige Teilnahme der Funktionäre an der Produktionsarbeit und enge Verbindung mit den Massen sollen dafür garantieren, daß die Revolutionskomitees ihren Rätecharakter bewahren (Näheres dazu Art. 11). Wie allerdings die Entwicklung seit Bildung der ersten Revolutionskomitees im Jahre 1968 zeigt, sind diese Ausschüsse im Begriffe, $\mathrm{zu}$ bloßen Transmissionsinstrumenten der lokalen Parteiausschüsse $\mathrm{zu}$ werden. Ähnlich wie die frührussischen Sowjets nach und nach „bolschewisiert" wurden, läßt sich in China eine "Parteiisierung" der eigentlich als Träger direkter Demokratie gedachten Revolutionskomitees beobachten ${ }^{14}$.

d) Das Selbstverwaltungsrecht der Minoritäten (Art. 24) wird zwar garantiert und soll durch Volkskongresse und Revolutionskomitees ausgeübt werden. Die Verfassung verurteilt jedoch gleichzeitig jede Form eines „lokalen Nationalismus" und erklärt Minderheitengebiete für "unabtrennbar" (Art. 4). Auch hier läßt sich eine beträchtliche Reduzierung der Bestimmungen gegenüber der alten Verfassung feststellen. War die Selbstverwaltungsmaterie für Gebiete nationaler Autonomie dort noch in 6 ausführlichen Bestimmungen geregelt (Art. 67-72), so genügt jetzt ein einziger Artikel! Die alte Dreiteilung in Autonome Gebiete, Autonome Bezirke und Autonome Kreise wurde beibehalten.

e) Stiefmütterlich sind Justiz und Staatsanwaltschaft geregelt. Anstelle der $12 \mathrm{Be}-$ stimmungen in der alten Verfassung (Art. 73-84) sind „Volksgerichte und Volksanwaltschaft" jetzt ebenfalls nur noch auf einen einzigen Artikel (25) beschränkt. Während der Kulturrevolution waren ja gerade die drei Sektoren Polizei, Justiz und Staatsanwaltschaft in den Beschuß der Revisionismuskritik geraten - und haben sich davon nie mehr erholt. Von Unabhängigkeit der Gerichte ist heute im Gegensatz zur alten Verfassung - nirgends mehr die Rede. Wichtige kriminelle und konterrevolutionäre Fälle sollen, wie es heißt, im Wege der Massendiskussion entschieden werden, wie überhaupt die „Massenlinie“ das gesamte Organisationswesen wie ein roter Faden durchzieht. Von der „Massenlinie“ im Gerichtsverfahren ist allerdings expressis verbis nicht die Rede.

f) Im neuen Verfassungstext überhaupt nicht erwähnt ist das Amt des Staatspräsidenten, dem in der alten Verfassung immerhin 8 Artikel (Art. 39-46) gewidmet waren. Mit dem Amt des Staatspräsidenten entfielen auch die beiden wichtigsten,

14 Näheres dazu Oskar Weggel, „Die Parteiausschüsse als Widersacher der Revolutionskomitees ${ }^{\alpha}$, Band 34 der Mitteilungen des Instituts für Asienkunde, Hamburg 1970. 
ihm früher zugeordneten Organe, nämlich die „Oberste Staatskonferenz“ und der "Nationale Verteidigungsrat". Es wurde auch kein Ersatz in Form eines kollektiven Staatspräsidiums vorgesehen, obwohl man eine solche Entwicklung im Zuge der allgemeinen Tendenz zur Kollektivführung hin eigentlich hätte erwarten können. Offensichtlich aber sind die Hypotheken Liu Shao-ch'is, die immer noch auf diesem Amte lasten, so schwer, daß man sich nicht einmal zu einer modifizierten Wiedereinführung der Position entschließen konnte. Statt dessen sind die Funktionen, die früher dem "Vorsitzenden der VR China“ zustanden, auf andere Gremien verteilt worden. Der „Oberbefehl über die Streitkräfte des Landes“ beispielsweise, der nach dem alten Artikel 42 dem Staatspräsidenten zukam, ist nunmehr auf den Vorsitzenden des $\mathrm{ZK}$ der $\mathrm{KPCh}$, also de facto auf Mao Tse-tung, übergegangen (Art. 15/II der neuen Verfassung). Die Ernennung der höchsten Staatsrepräsentanten obliegt nunmehr dem NVK (Art. 17). Die „Verkündigung“ der vom NVK beschlossenen Gesetze und Erlasse, die früher dem Staatspräsidenten oblag, wurde keinem anderen Organ expressis verbis zuerkannt. Dies war auch nicht nötig, da es seit Jahren keine formellen Gesetze in der VR China mehr gibt. Sollte die Notwendigkeit dafür eines Tages aber doch wieder auftauchen, so könnte der Ständige Ausschuß des Nationalen Volkskongresses einspringen, der ja offensichtlich überhaupt eine Art Auffangbecken für alle jene Kompetenzen bilden soll, die früher dem Staatspräsidenten zustanden. U. a. empfängt er ja beispielsweise ausländische Gesandte, genehmigt Verträge mit dem Ausland und macht sie rückgängig. (Im übrigen fungierte bis Januar 1975 in der Person des greisen Tung Pi-wu seit Jahren ein "Acting President“, der ebenfalls Gesandte empfing, Glückwunschschreiben bei staatlichen Anlässen entsandte und überhaupt wie ein Staatspräsident auftrat, ohne $\mathrm{da}$ allerdings diese Position konstitutionell formalisiert worden wäre!).

Das eigentliche Organ, das den Staatsapparat ähnlich bestimmt, wie die Seele den Körper, ist jedoch die Partei mit all ihren Unterorganisationen und Zellen. Während die KP 1954 nur in der Präambel erwähnt worden war, taucht sie in der neuen Verfassung - auch außerhalb der Präambel - gleich fünfmal auf, nämlich in Art. 2, 15, 16, 17 und 26. Wie vor allem aus Artikel 2 hervorgeht, steht die Partei über dem Staat. Auch bricht Parteirecht (die Parteisatzung von 1973) entgegenstehendes Verfassungsrecht. Wiederum über dem Parteirecht steht das „Mao-Tsetung-Denken" (Art. 2, 11).

Der Text ist im übrigen ein rein innenpolitisches Dokument, abgesehen von Artikel 18, der die Berechtigung zur Ratifizierung völkerrechtlicher Verträge regelt, taucht das Ausland nur in Art. 15 auf, wo die Feinde („Imperialismus, Sozio-Imperialismus und ihre Lakaien") enumeriert sind.

\section{Grundrechte und Grundpflichten}

Abschnitt III enthält die "Grundrechte und Grundpflichten „der Staatsbürger“. Von "Genossen“ ist nicht die Rede! Wie in der alten Verfassung sind hier die üblichen formalen Rechte (Wahlrecht, Freiheit der Meinung, der Vereinigung, der Demonstration, der Religion usw.) aufgezählt, deren relativer Wert vor allem während der Kulturrevolution deutlich geworden ist. Das „Recht zum Streiken“ (Art. 28) ist neu, ohne daß ihm freilich in der Praxis Bedeutung zukäme. Grundrechtsähnlich sind auch die in Art. 13 festgelegten Rechte der Massen, „frei zu sprechen, ihre Meinung voll zu äußern, große Debatten abzuhalten und Wandzei- 
tungen zu schreiben" (Art. 13). In Artikel 12 kommt eine grundpflichtähnliche Bestimmung zum Ausdruck, bei der allerdings der Klassencharakter besonders kräftig durchscheint. Danach hat das „Proletariat“ eine umfassende Diktatur über die Bourgeoisie im Überbaubereich auszuüben. Präzisiert worden ist gegenüber 1954 die Religionsfreiheit. Damals hieß es in Art. 88, daß jeder Bürger „Glaubensfreiheit genießt". Heute lautet Artikel 28 - ebenso übrigens wie die entsprechende Stelle im „Entwurf“: „Die Bürger der VR China ... haben das Recht, einer Religion anzugehören, sowie das Recht, keiner Religion anzuhängen. Auch ist es ihnen freigestellt, den Atheismus zu verkünden“. Besonders betont in der neuen Verfassung ist auch das Petitionsrecht jedes einzelnen gegenüber Staatsorganen auf sämtlichen Ebenen, sei es nun in schriftlicher oder mündlicher Form. Niemand ist berechtigt, ein solches Vorgehen zu verhindern. Im „Entwurf“ hatte es geheißen, daß die "fundamentalsten Rechte und Pflichten der Bürger darin bestehen, den Vorsitzenden Mao Tse-tung und seinen engen Waffengefährten, den stellvertretenden Vorsitzenden Lin Piao, zu unterstützen" (Art. 26). Diese Regelung ist revidiert worden, indem nun anstelle der beiden Personen der Ausdruck „Kommunistische Partei Chinas" gesetzt wurde (Art. 26/I). Auch hier also eine Versachlichung und Entpersonalisierung!

\section{Zusammenfassung:}

Während die neue, beim IV. Nationalen Volkskongreß eingesetzte Administrationsspitze personell "gemäßigt" ausgefallen ist, hat man der kulturrevolutionären Linken im Verfassungstext explizite Zugeständnisse gemacht - und dabei einem ihrer Exponenten bezeichnenderweise überdies das Privileg eingeräumt, die Änderungen im Verfassungstext vor dem NVK-Plenum zu erläutern.

Die neue Verfassung ist nicht mehr, wie ihre Vorgängerin von 1954, das Grundgesetz eines "neudemokratischen“, sondern vielmehr eines „sozialistischen" Staatswesens mit allen sich daraus ergebenden Konsequenzen. U. a. werden beispielsweise offiziell nur noch zwei - sozialistische - Eigentumsformen anerkannt.

Die neue Verfassung gleicht weitgehend dem kulturrevolutionären „Entwurf“ von 1970, ist allerdings von "Lin-Piao-Relikten“ gesäubert und „entpersonalisiert" worden (Streichung der Namen Mao Tse-tungs und Lin Piaos!).

Der Verfassungstext von 1975 ist wesentlich „transparenter“ als das 19 Jahre ältere Grundgesetz von 1954; Das „Mao-Tse-tung-Denken steht jetzt ganz oben an, gefolgt vom Parteirecht, das wiederum „Verfassungsrecht bricht“. Dies wird besonders deutlich in Artikel 2, wo es - ganz übereinstimmend mit der Diktion Mao Tse-tungs - heißt, „daß die Kommunistische Partei der führende Kern des ganzen chinesischen Volkes" ist und daß die Arbeiterklasse ihre Führung über den Staat „durch ihre Vorhut, die KPCh" ausübt. Außerdem wird die KP, die 1954 nur in der Präambel erwähnt war, im neuen Text gleich fünfmal angeführt.

Dreh- und Angelpunkt der Verfassung aber ist der dem ganzen Text zugrundeliegende Gedanke der "permanenten Revolution“, der den Text nicht nur im Sinne eines nachdrücklichen Klassenkampfpostulats einfärbt (Formulierung von Feindbildern innen- und außenpolitischer Art), sondern darüber hinaus auch für eine „kulturrevolutionäre" Sprachregelung sorgt (große Debatten, Wandzeitungen, Mitarbeit von Kadern in der Produktionsarbeit etc.) und dem permanenten Kampf gegen die Bürokratie förderlich ist (Verwaltungsvereinfachung, Reduzierung der juristischen Bestimmungen etc.). 
The Problem of Western Pattern in the Latest Discussion on Political Development

\section{By Manfred Mols}

Current theories of development suffer from a cultural bias: that western society a western performance of the economy, western styles of life etc. are the final ends of development. Looked at more closely one realizes that the western paradigm reveals some ambiguities. To a certain extent a complete reproduction of the West ist neither tolerable nor realizable. On the other hand, the western paradigm provides a series of experiences which deserve attention. First: students of development have been made sensitive for a difference between development and modernization, representing the latter a sort of take-off quality for development. Second: Development must be defined as the relative autonomy of goalsetting of a societal system, driven by what some people have called cultural dynamics. Third: The very core of the western developmental paradigm cannot consist of ideals in form of structural analogies, but of a set of qualities like capacity, differentiation, quality of life etc., which are abstract enough not to call for direct imitation in a sense of structural correspondence, qualities which would nevertheless direct the attention to intrinsic features of the western experience.

Given this body of general considerations with regard to his subject, the author tries in a second part of his essay to apply this to political development. Especially the three experiences are repeated referring now to what the developmental theories of political scientists might contribute. One of the by-products of this second part is a delineation of developmental thinking as well as its practical impacts both in economics and in political science.

\section{The New Constitution of the People's Republic of China}

\section{By Oskar Weggel}

While the persons whom the Fourth National People's Congress has placed in the top positions of the People's Republic of China's administration, are more or less on the "moderate" side, explicit concessions have been made, in the text of the Constitution, to the cultural revolutionary leftists. One of them was even granted the privilege of explaining the changes made in the revised text of the Constitution to the plenum of the National People's Congress.

Unlike its predecessor of 1954, the revised Constitution is not the basic law for a "new democracy" but for a "socialist" state, with all the consequences resulting from this change. One of those consequences is e. g. the fact that only two socialist - kinds of ownership of the means of production are officially recognized now.

The revised Constitution very much resembles the "cultural revolutionary draft" of 1970 but the present authors have eliminated all reminiscences of Lin Piao and all reference to persons, including the names of Mao Tse-tung and Lin Piao.

The text of the 1975 Constitution is much more "transparent" than its predecessor of 19 years ago. The item of foremost importance now is the "Mao Tse-tung 
Thought", followed by Party law which has precedence over constitutional law. This is revealed, with great clarity, in Article 2.

The crucial point of the revised Constitution, however, is the idea of "permanent revolution" on which the whole text is based. Not only have the authors emphasized the necessity of class struggle (against all enemies at home and abroad) but they also use "cultural revolutionary" language, referring to "great debates", "big-character posters", cadres participating in productive work etc., and encourage the permanent struggle against bureaucratic tendencies by simplifying the administration, reducing the codified rules of law in favour of the application of the mass line, etc.

\section{Parliamentary Democracy in Bangladesh - An Analysis of Constitutional State}

\section{By Horst Hartmann}

Bangladesh is one of the very few developing nations which have opted for parliamentary democracy after Independence. Obviously, the constitution-makers were influenced by the Indian "model" of a one-party-dominant-system, as they tried to stabilize the system by introducing special provisions against the desintegrative effects of partydissensions. However, it appears that the direction of constitutional development in Bangladesh is quite different from what was envisaged.

Both the law and order situation and economic conditions in Bangladesh have been very grave since it came into being. It is not for want of either legislative backing or administrative power that the government failed to cope with the threats to stability. As Prime Minister Sheikh Mujibur Rahman enjoys the unqualified support of the Awami League which in turn commands an overwhelming majority in the Parliament (Jayio Sangsad). There is no Opposition party able to provide a credible alternative national government. Indeed, the parliamentary Opposition is virtually dead - in contrast to the Indian situation. Last June the Government even enacted an amendment to the Special Powers Act authorising the government to arrest hoarders, blackmarketeers and smugglers. Hence, the Sheikh could not possibly claim that he lackes the power to restore economic distress and enforce law and order. On all accounts, the Sheikh did not act with determination and speed to tone up the administration and to combat starvation.

Sheik Mujibur Rahman has been Bangladesh's principal hope for stability and progress. However, as he has not produced results enough, the feeling of hopelessness and dispair has undermined his formerly undisputed Charisma. The dismissal of hundreds of government servants on charges of corruption in recent months is not known to have improved matters. The Sheikh has had reason to be disgusted with the politicians and their hangers-on who without doubt have abused their position to amass fortunes. But he has failed to exercise a healthy check on mismanagement and corruption, while using his special powers to repress opposition.

In fact, parliamentary democracy in Bangladesh was more formal than real from the very beginning of its existence. As it appears the disguised personal rule may 